\title{
The effects of conjugated linoleic acid isomers cis-9,trans-11 and trans-10,cis-12 on in vitro bovine embryo production and cryopreservation
}

\author{
V. A. Absalón-Medina, ${ }^{\star 1}$ S. J. Bedford-Guaus, † R. O. Gilbert,† L. C. Siqueira,‡ G. Esposito,§ A. Schneider,\# \\ S. H. Cheong, † and W. R. Butler*2 \\ ${ }^{*}$ College of Agriculture and Life Sciences, Animal Science Department, and \\ †College of Veterinary Medicine, Department of Clinical Sciences, Cornell University, Ithaca, NY 14853 \\ ¥Universidade Federal de Santa Maria, Santa Maria, RS 8690, Brazil \\ §Università degli Studi di Napoli Federico II, Portici, Napoli 80138, Italy \\ \#Universidade Federal de Pelotas, Pelotas, RS 96001, Brazil
}

\begin{abstract}
Conjugated linoleic acid (CLA) isomers can affect the lipid profile and signaling of cells and thereby alter their function. A total of 5,700 bovine oocytes were used in a structured series of experiments to test the effects of CLA cis-9,trans-11 and CLA trans-10,cis-12 in vitro. In experiment 1 , high doses of each CLA isomer during in vitro maturation (IVM) were compared with high or low doses during the entire in vitro culture (IVC) of parthenogenetic embryos. High doses of the CLA isomers ranged from 50 to $200 \mu M$ and low doses were 15 and $25 \mu \mathrm{M}$. In experiment 2 , the low doses of each CLA isomer were tested during IVM/IVC on embryos produced by in vitro fertilization (IVF). Experiment 3 compared the effects of $15 \mu M$ doses of each CLA isomer during IVM or IVC of IVF embryos. In experiment 4, post-rewarming survival rates and blastomere counts were assessed for embryos supplemented with each CLA isomer during IVM or for $36 \mathrm{~h}$ before vitrification. In experiment 1 , when either CLA isomer was provided only during IVM, we observed no effects on overall rates of maturation, cleavage, or blastocysts $(92.2 \pm 1.6 \%, 78.3 \pm 4.1 \%$, and $28.9 \pm 5.1 \%$, respectively). However, high doses of each CLA isomer, but not low doses, during the entire embryo culture period decreased blastocyst rates (5-20\%) in a dose-dependent manner. Cleavage rates improved with 15 or $50 \mu M$ CLA trans-10,cis-12. Progesterone concentrations in maturation media were significantly increased by high doses of each CLA isomer compared with control, but low doses of CLA isomers had no effect. In experiment 2 with IVF embryos, low doses of each CLA isomer did not alter cleavage rates (average $84.9 \pm 1.9 \%$ ) and only
\end{abstract}

\footnotetext{
Received November 13, 2013.

Accepted June 18, 2014.

${ }^{1}$ Current address: Department of Clinical Studies Section of Reproduction and Behavior, School of Veterinary Medicine, University of Pennsylvania New Bolton Center, Kennett Square 19348.

${ }^{2}$ Corresponding author: wrb2@cornell.edu
}

$25 \mu M$ CLA trans-10, cis-12 during IVC reduced blastocyst rates below those of controls $(25.5 \pm 2.1$ vs. 38.2 $\pm 2.3 \%)$. The lipid content of embryos was increased and relative expression of the BIRC5 (baculoviral IAP repeat containing 5) gene was depressed by CLA trans10,cis-12. In experiment 3 , low doses $(15 \mu M)$ of each CLA isomer during IVC significantly reduced blastocyst rates $(20.6 \pm 2.4 \%$ and $27.7 \pm 1.2 \%$ vs. $34.18 \pm$ $1.2 \%$ for CLA trans-10, cis-12 and CLA cis-9,trans- 11 compared with control, respectively) with less effect of each CLA during IVM. In experiment 4, adding 100 $\mu M$ CLA cis-9,trans-11 during the final $36 \mathrm{~h}$ of culture resulted in a high survival rate after rewarming and culture, and the higher embryo blastomere count was comparable to that of control embryos not undergoing vitrification. In conclusion, supplementation with either CLA isomer did not improve embryo production, but inclusion of CLA cis-9,trans-11 before vitrification improved the quality of bovine IVF embryos after rewarming and culture.

Key words: conjugated linoleic acid, bovine, embryo, cryopreservation

\section{INTRODUCTION}

Conjugated linoleic acid isomers comprise a group of PUFA derived from linoleic acid (C18:2n-6) during biohydrogenation by ruminal flora in cattle and sheep. Conjugated linoleic acid is also produced from $\Delta^{9}$ desaturation of vaccenic acid (trans-11 18:1; Bougnoux et al., 2010). Comparing the 2 primary CLA isomers, cis-9,trans-11 $(\boldsymbol{c 9} \mathbf{9}, \mathbf{t 1 1})$ is substantially more abundant than trans-10,cis-12 (t10,c12; Lock and Bauman, 2004; Bauman et al., 2008). These CLA isomers can affect the membrane lipid profile and cell signaling, thereby altering their function in vitro (Pereira et al., 2008; Al Darwich et al., 2010; Stinshoff et al., 2014). Regarding changes in lipid composition, CLA isomers become relevant for the improvement of some assisted reproduction technologies such as cryopreservation 
of gametes and embryos (Pereira et al., 2007, 2008; Pereira and Marques, 2008). Embryos may suffer considerable morphological and functional damage during cryopreservation and the extent of the damage, as well as differences in postthaw and rewarming performance, may be highly variable depending on the developmental stage and origin (i.e., in vitro-produced or in vivo-derived embryos). Currently, 2 methods exist for oocyte and embryo cryopreservation - slow freezing or vitrification - of which the latter seems to yield better results when working with relatively small numbers of embryos (Pereira and Marques, 2008). Cytoplasmic lipid content and lipid composition of membranes are of concern for in vitro embryo production because they can be affected by culture media in ways that increase their vulnerability to damage during cryopreservation procedures.

In regard to media composition, supplementation of embryo culture with serum may yield higher blastocyst rates; however, serum supplementation also increases cytoplasmic lipid uptake and lowers phosphatidyl choline and cholesterol esters (Pereira et al., 2007; Al Darwich et al., 2010). Excessive lipid accumulation may occur through increased lipid uptake from the medium or alterations of mitochondrial metabolism, resulting in reduced cryotolerance (Pereira and Marques, 2008). Serum-supplemented media can result in increased lipid accumulation and reduce bovine embryo developmental competence (Abe et al., 2002; Reis et al., 2003). Recent evidence indicates that excessive levels of FA in embryos impair mitochondrial activity and metabolism (Van Hoeck et al., 2013). Alternatively, when serum-containing culture medium was supplemented with CLA $t 10, c 12$, lipid droplet formation in bovine embryos was reduced (Pereira et al., 2007, 2008). Those authors proposed that embryo incorporation of CLA $t 10, c 12$ during culture increased membrane fluidity, thus conferring embryos with greater resistance to cryopreservation-induced damage. However, Al Darwich et al. (2010), using similar culture conditions, reported that CLA $t 10, c 12$ either decreased or had no benefit on post-rewarming survival rates in embryos following cryopreservation.

Among the functions of various PUFA, some have a role in cholesterol metabolism and the regulation of steroidogenesis (Fouladi-Nashta et al., 2009; Hughes et al., 2011). Along these lines, there is debate currently as to the effect of progesterone secretion by cumulus cells during oocyte maturation. Although some studies report that progesterone produced during this period negatively affects later embryo development, others suggest that this hormone is involved in the acquisition of oocyte competence and regulation of cumulus-cell apoptosis associated with the defined switch from estra- diol (E2) dominance to progesterone (P4) dominance in preovulatory follicles (reviewed by Fair and Lonergan, 2012).

Polyunsaturated fatty acids, including CLA isomers, have various effects on cells depending on tissue type and, as noted above, contradictory results have been reported for their potential effects on embryo development. Given the findings among previous studies on cell membranes and lipid content, the potential benefits of CLA supplementation during in vitro culture (IVC) of bovine oocytes and embryos require more systematic investigation. In the current series of experiments, we evaluated the effects of supplementing culture medium with varying concentrations of 2 CLA isomers for their effects upon bovine oocytes, parthenotes, and preimplantation in vitro fertilized (IVF) embryos. In addition, the effects of CLA supplementation on postrewarming survival and quality of vitrified embryos were assessed.

\section{MATERIALS AND METHODS}

\section{Chemicals and Preparation of BSA-CLA Complexes}

All chemicals and reagents were obtained from SigmaAldrich Co. (St. Louis, MO), unless otherwise stated. The CLA isomers cis-9,trans-11 and trans-10,cis-12 (>90\% pure) were purchased from Nu-Chek Prep Inc. (Elysian, MN). The CLA isomers were conjugated to FA-free BSA in a 4:1 ratio to prepare $4.5 \mathrm{~m} M$ BSACLA stocks using a method adapted from Keating et al. (2006). Subsequent dilutions were made in culture medium.

\section{Experimental Design}

This study was structured in 4 experiments. In experiment 1 , the effects of $c 9, t 11$ and $t 10, c 12$ were tested at high $(50,100$, and $200 \mu M)$ and low $(15$ and $25 \mu M)$ concentrations during in vitro production of embryos after parthenogenetic activation. In experiment 2 , the effect of each CLA isomer at low concentrations was tested during in vitro production of IVF embryos. In experiment 3, the effect of each CLA isomer at $15 \mu M$ was tested on IVF embryos during in vitro maturation (IVM) only or during embryo culture. Finally, in experiment 4, the effects of short-term exposure of each CLA isomer was tested on IVF embryos during IVM $(15 \mu M)$ or at $100 \mu M$ for the final $36 \mathrm{~h}$ of culture before embryos were vitrified for cryopreservation and evaluated after rewarming.

Experiment 1. Experiment 1 studied the effects of CLA supplementation before and after parthenogenetic activation of bovine oocytes on embryonic development. 
The aims of this experiment were to compare the effect of high-dose CLA supplementation in maturation medium only with high- or low-dose CLA supplementation throughout the entire embryo culture period. Oocytes were activated by parthenogenesis to allow direct tests for CLA isomer effects on embryo development without potential confounding effects on sperm or fertilization. Dose ranges for CLA isomers were based on Pereira et al. (2008), and both CLA isomers $(c 9, t 11$ and $t 10, c 12)$ were tested; 50, 100, and $200 \mu M$ doses were considered high and 15 and $25 \mu M$ doses were considered low. For the low-dose trial, a third group (positive control; 50 $\mu M)$ was added to allow comparison with the effects of previous high doses. Osmolality (mOsm) of culture media was similar among all CLA treatment groups with carrier FA-free BSA added to each control group. No mineral oil overlay was used for maturation wells. The 3 component trials in this experiment were as follows: the evaluation of CLA supplementation during IVM (n = 738 oocytes); high-dose CLA supplementation throughout oocyte and embryo culture $(\mathrm{n}=1,514$ oocytes); and low-dose CLA-supplementation throughout culture ( $\mathrm{n}=1,206$ oocytes). Each component part was replicated 3 or more times. Following oocyte removal, IVM media from each treatment group were stored at $-20^{\circ} \mathrm{C}$ for progesterone analysis.

Experiment 2. Experiment 2 studied the effects of low-dose CLA supplementation throughout the entire culture period before and after in vitro fertilization (IVF). In 4 replicates, a total of 839 oocytes were matured and fertilized, and the corresponding embryos were cultured in vitro. Oocytes were placed in medium supplemented with CLA or carrier control for the entire IVC period, including IVM. Treatment groups were 15 and $25 \mu M$ doses for each CLA isomer $(c 9, t 11$ and $t 10, c 12$ ) and the corresponding carrier control. Variables measured included cleavage and blastocyst formation rates, as well as the neutral lipid (hereafter, lipid) content of embryos at d 8 postfertilization assessed by Nile red fluorescent staining under an epifluorescent microscope (Bonilla and Hansen, 2009). For all experiments involving staining, fixed embryos were mounted on fluorescent antibody microslides with 2 etched 10 -mm-diameter circles surrounded by white ceramic ink (Thermo Scientific, Fair Lawn, NJ). Relative mRNA expression of the BIRC5 and HSPA1A genes (embryonic survival markers baculoviral IAP repeat containing 5 and heat shock $70 \mathrm{kDa}$ protein $1 \mathrm{~A}$, respectively) was measured on d 8 after IVF by reverse transcription quantitative PCR (RT-qPCR) based on Rief et al. (2002) and Rizos et al. (2004).

Experiment 3. Experiment 3 studied the effects of low-dose CLA supplementation during oocyte maturation only or during embryo culture following IVF. The effects of a low $(15 \mu M)$ dose of the tested CLA isomers were evaluated either during IVM only or following IVF. In 4 replicates, 902 oocytes were used to assess embryonic viability at $\mathrm{d} 8$ postfertilization. Variables measured were cleavage and blastocyst rates and total blastomere count, assessed using Hoechst 33342 staining (Bonilla and Hansen, 2009).

Experiment 4. Experiment 4 studied the effects of CLA supplementation on embryo viability following vitrification and rewarming. Based on the results of the previous experiments, the focus of this final experiment shifted to short-term treatment with CLA isomers on embryos undergoing cryopreservation. In 2 replicates, 502 oocytes were used across 6 groups including control, $c 9, t 11$ (15 $\mu M$ IVM or $100 \mu M$ IVC), and $t 10, c 12$ (15 $\mu M$ IVM or $100 \mu M$ IVC). The sixth group was a quality control without CLA supplementation and not undergoing vitrification procedures (Control+). Two groups received $15 \mu \mathrm{M}$ CLA only during IVM and remained CLA-free throughout subsequent culture. Two groups were treated late in culture beginning at d 6.5 postfertilization with $100 \mu M t 10, c 12$ or $c 9, t 11$ isomers for $36 \mathrm{~h}$ before vitrification. On d 8 postfertilization, embryos $(\mathrm{n}=180$ total across 6 groups) were vitrified (see Vitrification and Rewarming section). One hour later, embryos were rewarmed and incubated for $24 \mathrm{~h}$, fixed, and stained with Hoechst 33342 to count blastomeres. Variables measured included cleavage, blastocyst and post-rewarming blastocyst survival rates, as well as total blastomere cell counts. Survival rates were assessed as the original number of embryos being vitrified that subsequently re-expanded or developed (hatched) during the post-rewarming culture period.

\section{Oocyte Recovery and Selection}

Bovine ovaries were collected at an abattoir and transported $(120 \mathrm{~km})$ to the laboratory in prewarmed $\left(30-35^{\circ} \mathrm{C}\right)$ lactate Ringer's solution. Cumulus-oocyte complexes (COC) from 2- to 8-mm follicles were aspirated with an 18-gauge hypodermic needle attached to an aspiration pump unit adjusted to a pressure of 22.5 $\pm 2.5 \mathrm{~mL}$ of $\mathrm{H}_{2} \mathrm{O}$ per minute. Follicular fluid supernatant was removed and the pellet containing COC was transferred to a $15-\mathrm{mL}$ tube, where it was resuspended with holding medium. The contents were then poured gently into a $100-\mathrm{mm}$ petri dish. Holding medium consisted of TCM-199 Hanks' salts (Invitrogen, Grand Island, NY) supplemented with $10 \%$ fetal calf serum (FCS; Invitrogen), $25 \mu \mathrm{g} / \mathrm{mL}$ of gentamicin, and $5 \mu \mathrm{g} /$ $\mathrm{mL}$ heparin, at $\mathrm{pH} 7.35 \pm 0.02$ and $300 \pm 1 \mathrm{mOsm}$. After morphological assessment of COC, only those with several layers of granulosa cumulus cells and oocytes with a homogeneous cytoplasm were selected for use. 
The entire process was performed within $5 \mathrm{~h}$, including transportation.

\section{IVM}

Selected COC were matured in groups of $40 \pm 5$ for $23 \pm 1 \mathrm{~h}$ in $400 \mu \mathrm{L}$ of TCM-199 (Earle's salts) enriched with $10 \% \mathrm{FCS}, 22 \mu \mathrm{g} / \mathrm{mL}$ sodium pyruvate, $1 \mathrm{mM}$ alanyl-glutamine, $0.1 \mathrm{~m} M$ taurine, $0.1 \mathrm{~m} M$ cysteamine, 1 $\mu \mathrm{g} / \mathrm{mL}$ estradiol, $5 \mu \mathrm{g} / \mathrm{mL} \mathrm{LH}$ (Sioux Biochemical Inc., Sioux Center, IA), $0.5 \mu \mathrm{g} / \mathrm{ml} \mathrm{FSH} \mathrm{(Sioux),} 10 \mathrm{ng} / \mathrm{mL}$ epidermal growth factor (BD Biosciences/Discovery Labware, Franklin Lakes, NJ), and $20 \mu \mathrm{g} / \mathrm{mL}$ gentamicin, at $\mathrm{pH} 7.35 \pm 0.02$ and $300 \pm 2 \mathrm{mOsm}$, and covered with light mineral oil (except for experiment 1) in a humidified atmosphere at $38.5^{\circ} \mathrm{C}$ with $5 \% \mathrm{CO}_{2}$ in air.

\section{Parthenogenetic Oocyte Activation and Embryo Culture After Maturation}

Parthenotes were obtained following the protocol used by Bastos et al. (2008). Briefly, groups of COC were placed into a small petri dish containing $0.2 \mathrm{mg}$ of hyaluronidase per $\mathrm{mL}$ of holding media $(\mathrm{pH} 7.35 \pm 0.02$, $300 \pm 2 \mathrm{mOsm}$ ) for $3 \mathrm{~min}$ and then transferred into a $1.8-\mathrm{mL}$ centrifuge tube. Contents were vortexed at maximum speed for $5 \mathrm{~min}$ to remove the cumulus cells. Cumulus-free oocytes were then resuspended in holding medium and, by fine glass pipette rotation, only those presenting a visible polar body indicating successful progression of nuclear maturation to the metaphase II stage (MII) were selected for parthenogenetic activation. Then, metaphase II oocytes were exposed to $5 \mu M$ ionomycin for 5 min in M-199 (Hanks' salts; Invitrogen) medium supplemented with $0.4 \%$ (wt/vol) BSA, rinsed 3 times in the same medium without ionomycin, and then incubated for $5 \mathrm{~h}$ in modified synthetic oviduct fluid medium (SOF, pH $7.4 \pm 0.01,275 \pm 5$ mOsm; Holm et al., 1999) containing $2.8 \mathrm{~m} M$ 6-dimethylaminopurine (6-DMAP; Susko-Parrish et al., 1994). After this short incubation treatment, oocytes were rinsed twice and cultured in SOF medium supplemented with $0.4 \%$ (wt/vol) BSA and both EAA and NEAA at $38.5^{\circ} \mathrm{C}$ in $5 \% \mathrm{CO}_{2}, 5 \% \mathrm{O}_{2}$, and $90 \% \mathrm{~N}_{2}$. Rates of cleavage were evaluated at $48 \mathrm{~h}$ after activation. After cleavage assessment, parthenogenetically activated embryos were cultured in groups of 10 in $100-\mu \mathrm{L}$ SOF droplets and transferred every $2 \mathrm{~d}$ to fresh medium. Blastocyst rates were assessed at $\mathrm{d} 8$ after activation.

\section{IVF}

Presumptive matured oocytes were transferred to a modified IVF medium (Fert-TALP; Parrish et al.,
1988) supplemented with $0.5 \mathrm{~m} M$ fructose, $6 \mathrm{mg} / \mathrm{mL}$ BSA FFA Fraction V, $30 \mu M$ penicillamine, $15 \mu M$ hypotaurine, $1.5 \mu \mathrm{M}$ epinephrine, $22 \mu \mathrm{g} / \mathrm{mL}$ heparin, 20 $\mu \mathrm{g} / \mathrm{mL}$ gentamicin, $\mathrm{pH}$ of $7.38 \pm 0.01,290 \pm 2 \mathrm{mOsm}$, and covered with light mineral oil in a humidified atmosphere at $38.5^{\circ} \mathrm{C}$ with $5 \% \mathrm{CO}_{2}$ in air for 18 to 22 h. For all the IVF experiments, frozen semen straws (Genex Cooperative Inc., Ithaca NY) from the same ejaculate of a single bull were thawed at $37^{\circ} \mathrm{C}$ for 30 s. Motile sperm were separated from nonmotile sperm, cryoprotectant, and debris by Percoll double density gradient $(90,45 \%)$ centrifugation at $700 \times g$ for 20 min. Subsequently, sperm were washed twice in $5 \mathrm{~mL}$ of TL-Semen $(\mathrm{pH} 7.39 \pm 0.01,295 \pm 2 \mathrm{mOsm})$ modified Tyrode's (Parrish et al., 1989) at $300 \times g$ for 5 min to remove Percoll. Finally, sperm were adjusted to a final concentration of $1.5 \times 10^{6} \mathrm{sperm} / \mathrm{mL}$ using Fert-TALP medium.

\section{In Vitro Embryo Culture}

A modified SOF medium (Holm et al., 1999) was used for in vitro embryo culture (IVC, $20 \mu \mathrm{g} / \mathrm{mL}$ gentamicin, $\mathrm{pH} 7.4 \pm 0.01,275 \pm 5 \mathrm{mOsm})$. After fertilization, putative zygotes were denuded at maximum vortex speed for $120 \mathrm{~s}$ and transferred to a modified SOF $\left(\mathrm{SOF}_{\text {early }}\right)$ supplemented with $10 \mu M$ EDTA, $0.5 \mathrm{~m} M$ fructose, $0.4 \%$ (wt/vol) BSA FFA Fraction V, and 0.1 $\mathrm{m} M$ taurine, and then placed in light mineral oil-covered droplets in a humidified atmosphere at $38.5^{\circ} \mathrm{C}$ with $5 \%$ $\mathrm{CO}_{2}, 5 \% \mathrm{O}_{2}$, and $90 \% \mathrm{~N}_{2}$. Following approximately 48 $\mathrm{h}$ in culture, cleavage rates were assessed and embryos were transferred to new droplets containing $\mathrm{SOF}_{\text {mid }}$, with EAA and NEAA, 0.4\% (wt/vol) BSA FFA Fraction $\mathrm{V}$, and $0.5 \mathrm{~m} M$ glucose in a humidified atmosphere at $38.5^{\circ} \mathrm{C}$ with $5 \% \mathrm{CO}_{2}, 5 \% \mathrm{O}_{2}$, and $90 \% \mathrm{~N}_{2}$. After approximately $96 \mathrm{~h}$, embryos were again transferred to fresh $\mathrm{SOF}_{\text {mid }}$ droplets under the same conditions. Finally, d 7 embryos were transferred for the last $24 \mathrm{~h}$ of culture to $\mathrm{SOF}_{\text {late}}$, which was $\mathrm{SOF}$ supplemented with $5 \%$ (vol/vol) FCS, $0.1 \mathrm{~m} M$ taurine, and $0.5 \mathrm{~m} M$ glucose in a humidified atmosphere at $38.5^{\circ} \mathrm{C}$ with $5 \% \mathrm{CO}_{2}, 5 \%$ $\mathrm{O}_{2}$, and $90 \% \mathrm{~N}_{2}$. Blastocyst rates were calculated as percentages of the original oocyte number.

\section{$R T-q P C R$}

For total RNA extraction, $100 \mu \mathrm{L}$ of Qiazol (Qiagen, Valencia, CA) was added to a batch of embryos (13 \pm 4 embryos) from each treatment group $8 \mathrm{~d}$ after fertilization, vortexed, and stored at $-80^{\circ} \mathrm{C}$. Total RNA was isolated and purified using the RNeasy Mini columns (Qiagen) and on-column RNase-free DNase treatment (Qiagen) following the manufacturer's instructions. 
Then, RNA was eluted in a final volume of $15 \mu \mathrm{L}$. The reverse transcription reactions were performed using a High Capacity cDNA Reverse Transcription Kit with RNase Inhibitor (Applied Biosystems, Foster City, CA) in a final volume of $20 \mu \mathrm{L}$. The RT-qPCR was performed using SYBR Green dye to evaluate RPS9 (reference gene), BIRC5, and HSPA1A relative mRNA expression with primers listed in Table 1 . The final amount of cDNA from each batch was diluted to allow the equivalent of cDNA for one embryo in each qPCR reaction. The PCR reactions were performed in duplicate in a $25-\mu \mathrm{L}$ final volume using Power SYBR Green Mastermix (Applied Biosystems) and $5 \mu \mathrm{L}$ of the diluted cDNA. The PCR reactions and fluorescence detection were performed in the ABI Prism 7300 Sequence Detector (Applied Biosystems). For each assay, 40 PCR cycles were run and a dissociation curve was included to verify the amplification of a single PCR product. Analyses of amplification plots were performed with the Sequence Detection Software (Applied Biosystems) to determine counts for each sample. Each assay plate contained negative controls and a standard curve with 5 diluted liver samples (serial dilutions with known RNA concentrations) to determine amplification efficiency of the respective primer pair, which was $1.13,1.29$, and 1.29 for RPS9, HSPA $1 A$, and BIRC5, respectively. The relative transcript expression was calculated using the $2^{-\Delta \Delta \mathrm{CT}}$ (cycle threshold) method (Livak and Schmittgen, 2001), using $R S P 9$ as a reference gene and the control group to correct the fold changes. The average intraassay coefficients of variation were $1.8,1.0$, and $0.9 \%$, for RPS9, HSPA1A, and BIRC5, respectively.

\section{Vitrification and Rewarming of Embryos for Evaluation}

Bovine embryos were vitrified (BoviPRO Vit Kit; Minitube, Verona, WI) according to the manufacturer's instructions. Briefly, treatment groups were placed separately in holding media. Embryos were exposed for $5 \mathrm{~min}$ to solution A followed by another $5 \mathrm{~min}$ in solution B. Subsequently, embryos were placed into the vitrification solution for no more than 1 min while being loaded into a semen straw. Loaded embryos spent 1 min in liquid $\mathrm{N}_{2}$ vapor before being plunged into liquid $\mathrm{N}_{2}$. After $1 \mathrm{~h}$, the embryos were rewarmed. For rewarming, straws were exposed to air temperature for $10 \mathrm{~s}$ followed by submersion in water at room temperature. Straws were flicked several times to combine the vitrification medium with the dilution solution. Embryos remained in the straws for $6 \mathrm{~min}$ at room temperature while rewarming. All embryos were cultured further for $24 \mathrm{~h}$ in $\mathrm{SOF}_{\text {late }}$ medium for final assessment of survival rates.

\section{Image Acquisition of Stained Embryos}

All slides were examined using an epifluorescent microscope (Imager Z1; Carl Zeiss Inc., Thornwood, NY) under a $20 \times 0.5$ NA ECPlan Neofluor objective (Carl Zeiss Inc.). Embryo samples were excited at 340 and $488 \mathrm{~nm}$ with emission of 470 and $515 \mathrm{~nm}$, to visualize DAPI (4',6-diamidino-2-phenylindole) nuclear stain and Nile red, respectively. With these excitation and emission wavelengths, Nile red stain accounted mostly for neutral lipids (i.e., triglycerides). Images were captured with a cooled charged-coupled device camera (AxioCam MRm; Carl Zeiss Inc.) and processed using AxioVision software (version 4.7.2; Carl Zeiss Inc.). Mean pixel intensity was reported in arbitrary units.

\section{Progesterone Radioimmunoassay}

Progesterone concentrations in IVM media samples were quantified by RIA (Elrod and Butler, 1993) with the addition of control medium to standards. Inter- and intraassay coefficients of variation were 5.35 and $5.54 \%$, respectively.

Table 1. Primers $(\mathrm{F}=$ forward; $\mathrm{R}=$ reverse $)$ used for real-time PCR

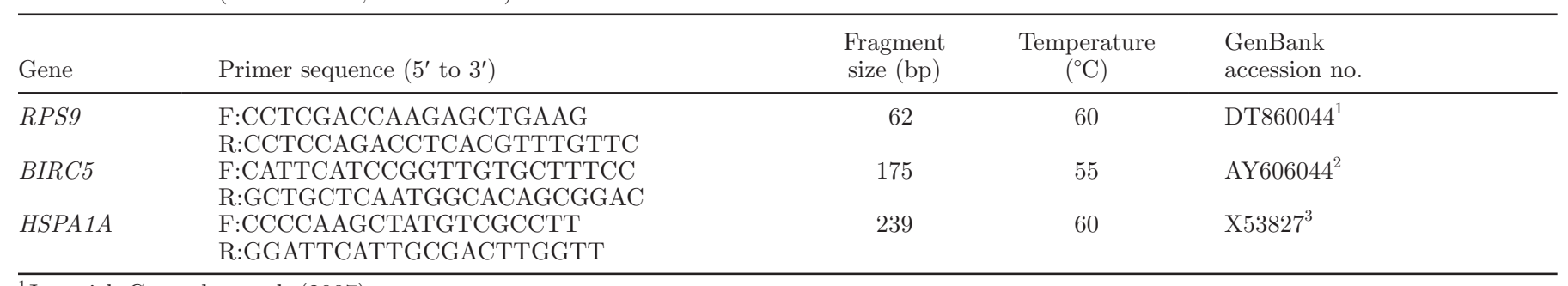

${ }^{1}$ Janovick-Guretzky et al. (2007).

${ }^{2}$ Rizos et al. (2004).

${ }^{3}$ Rief et al. (2002). 


\section{Statistical Analysis}

One-way ANOVA between treatments was performed using JMP version 10 (SAS Institute Inc., Cary, NC). For all experiments, oocyte groups were the experimental units. If replicates were different over time, we included replicate in our models as a random variable to control for the differences between and within experiments. If the main effect was significant, all means were compared using Tukey Honestly Significant Difference (HSD) test. A probability of $P<0.05$ was considered statistically significant and $P \leq 0.1$ was considered a trend. Proportional data not being normally distributed were arc-sine transformed, and results are presented in the tables as back-transformed data. Likewise, numerical data not normally distributed were transformed to natural logarithm for analyses.

\section{RESULTS}

\section{Experiment 1}

Supplementation with CLA during IVM did not significantly $(P>0.05)$ affect the rate of oocyte maturation or the proportion of cleaved embryos and blastocysts at any dose level of either CLA isomer (Table 2). Progesterone analyses of IVM media showed increased levels of progesterone in response to all high doses of each CLA isomer, and this effect was directly proportional to the CLA concentration (main effect $P<0.05$; Figure 1). Low CLA doses had no significant effect on progesterone levels among groups $(0.54 \pm 0.04,0.67 \pm$ $0.09,0.58 \pm 0.03,0.66 \pm 0.06$, and $0.62 \pm 0.1 \mathrm{ng} / \mathrm{mL}$ for control; t10,c12 $15 \mu M$; t10,c12 $25 \mu M$; $c 9, t 1115$ $\mu M$; and $c 9, t 1125 \mu M$, respectively).

Results of high-dose CLA supplementation (50, 100, and $200 \mu M$ for each isomer) throughout culture are summarized in Table 3. At $200 \mu M, t 10, c 12$ reduced maturation rates compared with the control group $(P<$ 0.05). Conversely, supplementation with $50 \mu M t 10, c 12$ improved cleavage rates compared with the control group $(P<0.05)$. Blastocyst rates were depressed by $t 10, c 12$ and $c 9, t 11$ isomers in a dose-dependent manner $(P<0.05)$.

The effects of low doses of CLA supplementation (15 and $25 \mu M$ for each isomer) for the entire culture period are presented in Table 4 . No significant differences were observed between groups for maturation rates. The group supplemented with $t 10, c 12$ at $15 \mu M$ showed the highest cleavage rate $(86.6 \%)$, which was higher $(P<$ $0.05)$ than that of the control group. Conversely, no statistical differences were detected among groups for blastocyst rates.

\section{Experiment 2}

In experiment 2, we tested the low concentrations of the $t 10, c 12$ and $c 9, t 11$ isomers from experiment 1 (Table 5 ). No differences were observed in cleavage rates; however, blastocyst rates were significantly depressed by $25 \mu M t 10, c 12$ supplementation. The lipid content of d 8 embryos was determined by epifluorescence microscopy after Nile red staining (Figure 2). Lipid content was increased by both 15 and $25 \mu M$ doses of $t 10, c 12$ compared with the control group $(P<0.05)$; treatment with $c 9, t 11$ had a significant effect only at 15 $\mu M$. Relative transcript expression of the BIRC5 gene was depressed by 15 and $25 \mu M t 10, c 12$ and by $15 \mu M$ $c 9, t 11$ (Figure 3). Transcript expression of HSPA1A was decreased only by $t 10, c 12$ at $15 \mu M$.

\section{Experiment 3}

Supplementation with low doses of each CLA isomer at different points throughout the embryo production process did not affect cleavage rates among groups $(P>0.05 ;$ Table 6$)$. However, blastocyst rates were depressed $(P<0.05)$ when $t 10, c 12$ was supplemented both during IVM and during the post-IVF culture period. Isomer $c 9, t 11$ affected blastocyst rate only when provided during IVC. Even with lower blastocyst rates

Table 2. Effects of high-dose CLA supplementation in maturation medium upon parthenogenetically activated embryo development (experiment 1$)^{1}$

\begin{tabular}{lcccc}
\hline Group & $\begin{array}{c}\text { Oocytes/ } \\
\text { group }\end{array}$ & $\begin{array}{c}\text { Maturation } \\
\text { rate }(\%)\end{array}$ & $\begin{array}{c}\text { Cleavage } \\
\text { rate }(\%)\end{array}$ & $\begin{array}{c}\text { Blastocyst } \\
\text { rate }^{2}(\%)\end{array}$ \\
\hline Control & $35.0 \pm 3.0$ & $94.0 \pm 2.0$ & $83.7 \pm 2.5$ & $31.5 \pm 6.2$ \\
trans-10, cis-12, $50 \mu M$ & $35.7 \pm 2.7$ & $90.9 \pm 5.1$ & $81.6 \pm 6.2$ & $25.2 \pm 8.1$ \\
trans-10, cis-12, $100 \mu M$ & $35.3 \pm 0.3$ & $90.5 \pm 2.6$ & $80.2 \pm 6.6$ & $29.5 \pm 1.3$ \\
trans-10, cis-12, $200 \mu M$ & $35.3 \pm 2.0$ & $88.9 \pm 2.5$ & $76.3 \pm 7.7$ & $33.4 \pm 10.5$ \\
cis-9,trans-11, $50 \mu M$ & $34.7 \pm 1.9$ & $93.3 \pm 0.7$ & $85.4 \pm 4.3$ & $22.9 \pm 4.7$ \\
cis-9,trans-11, $100 \mu M$ & $35.0 \pm 1.0$ & $94.2 \pm 2.9$ & $86.3 \pm 6.8$ & $28.5 \pm 7.0$ \\
cis-9,trans-11, $200 \mu M$ & $35.0 \pm 0.6$ & $89.6 \pm 1.7$ & $78.3 \pm 3.5$ & $31.1 \pm 9.1$ \\
\hline
\end{tabular}

${ }^{1}$ Results are means \pm SEM with 3 replicates. Means within columns were not significantly different.

${ }^{2}$ Blastocyst rates are based on percentages of the original oocyte number. 
Table 3. Effects of high-dose CLA supplementation during the entire culture period (pre- and post-activation) upon parthenogenetically activated embryo development (experiment 1$)^{1}$

\begin{tabular}{|c|c|c|c|c|}
\hline Group & $\begin{array}{l}\text { Oocytes/ } \\
\text { group }\end{array}$ & $\begin{array}{c}\text { Maturation } \\
\text { rate (\%) }\end{array}$ & $\begin{array}{l}\text { Cleavage } \\
\text { rate }(\%)\end{array}$ & $\begin{array}{l}\text { Blastocyst } \\
\operatorname{rate}^{2}(\%)\end{array}$ \\
\hline Control & $35.7 \pm 1.1$ & $94.4 \pm 1.5^{\mathrm{abc}}$ & $76.2 \pm 2.6^{\mathrm{bc}}$ & $28.0 \pm 4.2^{\mathrm{a}}$ \\
\hline trans-10,cis-12, $50 \mu M$ & $35.7 \pm 1.1$ & $97.1 \pm 1.1^{\mathrm{a}}$ & $87.2 \pm 2.8^{\mathrm{a}}$ & $17.9 \pm 1.8^{\mathrm{bc}}$ \\
\hline trans-10, cis- $12,100 \mu M$ & $36.2 \pm 1.1$ & $95.4 \pm 1.0^{\mathrm{ab}}$ & $80.9 \pm 2.2^{\mathrm{ab}}$ & $11.5 \pm 2.1^{\mathrm{cd}}$ \\
\hline trans-10,cis-12, $200 \mu M$ & $37.0 \pm 2.5$ & $90.1 \pm 1.5^{\mathrm{d}}$ & $76.1 \pm 3.4^{\mathrm{bc}}$ & $7.8 \pm 0.9^{\mathrm{d}}$ \\
\hline cis-9,trans-11, $50 \mu M$ & $35.8 \pm 1.4$ & $91.2 \pm 1.6^{\mathrm{cd}}$ & $71.5 \pm 3.9^{\mathrm{c}}$ & $23.3 \pm 2.2^{\mathrm{ab}}$ \\
\hline cis-9,trans-11, $100 \mu M$ & $35.3 \pm 1.6$ & $91.3 \pm 1.6^{\mathrm{cd}}$ & $77.1 \pm 3.4^{\mathrm{bc}}$ & $16.4 \pm 1.5^{\mathrm{c}}$ \\
\hline cis-9,trans-11, $200 \mu M$ & $36.7 \pm 1.5$ & $92.2 \pm 1.2^{\mathrm{bcd}}$ & $80.6 \pm 3.8^{\mathrm{abc}}$ & $15.2 \pm 1.2^{\mathrm{c}}$ \\
\hline
\end{tabular}

$\overline{\mathrm{a}-\mathrm{d}}$ Means within a column not sharing the same letter are significantly different $(P<0.05)$.

${ }^{1}$ Results are means \pm SEM with 6 replicates.

${ }^{2}$ Blastocyst rates are based on percentages of the original oocyte number.

Table 4. Effects of low-dose CLA supplementation during the entire culture period (pre- and postactivation) on parthenogenetically activated embryo development (experiment 1$)^{1}$

\begin{tabular}{lcccc}
\hline Group & $\begin{array}{c}\text { Oocytes/ } \\
\text { group }\end{array}$ & $\begin{array}{c}\text { Maturation } \\
\text { rate (\%) }\end{array}$ & $\begin{array}{c}\text { Cleavage } \\
\text { rate (\%) }\end{array}$ & $\begin{array}{c}\text { Blastocyst } \\
\text { rate }^{2}(\%)\end{array}$ \\
\hline Control & $34.2 \pm 1.0$ & $89.0 \pm 1.2$ & $67.7 \pm 2.8^{\mathrm{c}}$ & $20.7 \pm 1.9$ \\
trans-10, cis-12, $15 \mu M$ & $35.2 \pm 1.6$ & $94.5 \pm 1.8$ & $86.6 \pm 2.0^{\mathrm{a}}$ & $19.4 \pm 1.0$ \\
trans-10, cis-12, $25 \mu M$ & $33.8 \pm 0.6$ & $93.0 \pm 2.4$ & $75.5 \pm 3.2^{\mathrm{abc}}$ & $18.7 \pm 4.7$ \\
cis-9, trans-11, $15 \mu M$ & $33.6 \pm 1.4$ & $92.7 \pm 2.4$ & $79.2 \pm 4.1^{\mathrm{ab}}$ & $17.9 \pm 3.2$ \\
cis-9, trans-11, $25 \mu M$ & $35.0 \pm 1.1$ & $92.0 \pm 1.6$ & $69.9 \pm 3.8^{\mathrm{bc}}$ & $18.7 \pm 1.9$ \\
\hline
\end{tabular}

${ }^{\mathrm{a}-\mathrm{c}}$ Means within a column not sharing the same letter are significantly different $(P<0.05)$.

${ }^{1}$ Results are means \pm SEM with 5 replicates.

${ }^{2}$ Blastocyst rates are based on percentages of the original oocyte number.

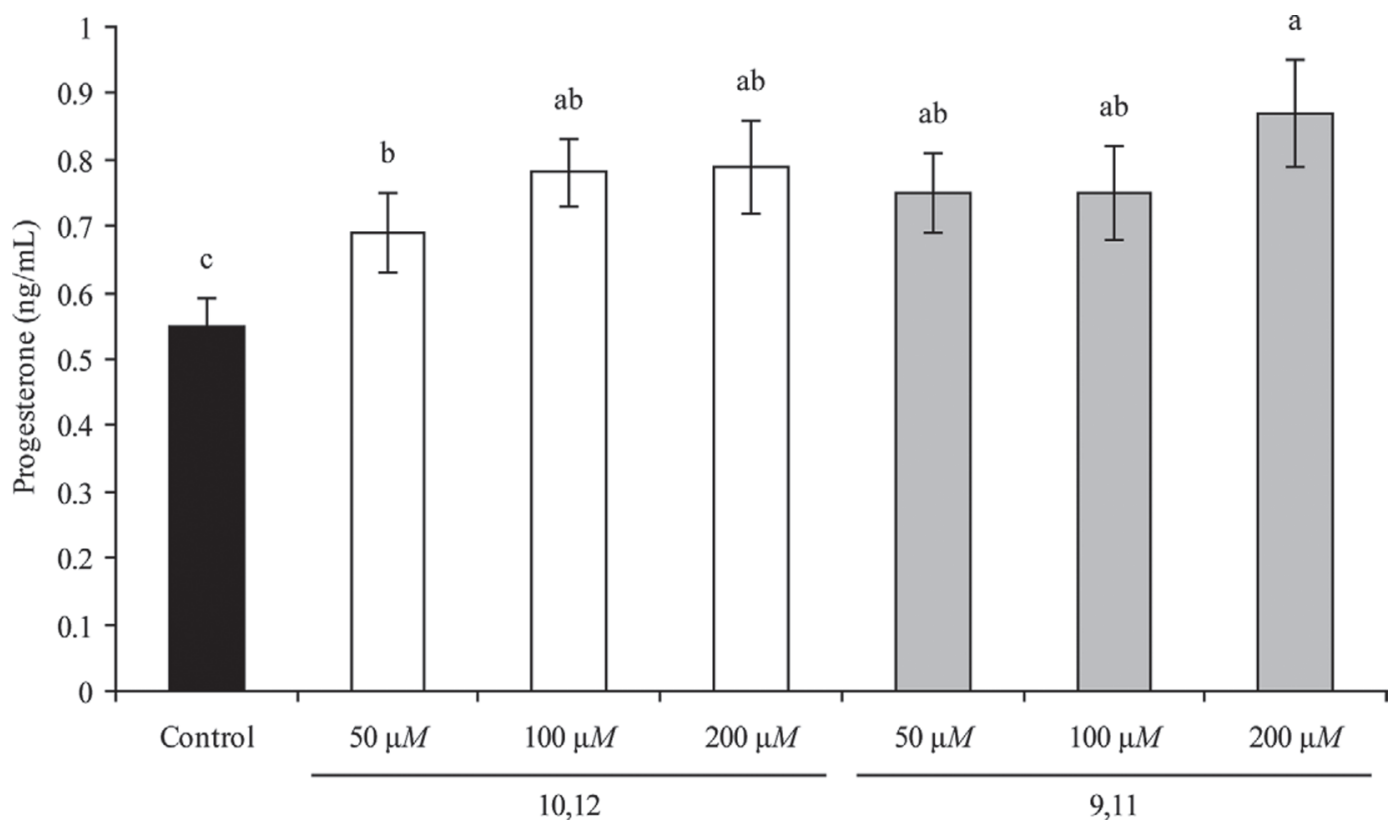

Figure 1. Effect of high doses of CLA isomers during oocyte maturation on progesterone concentrations in the medium (experiment 1). 10,12 = CLA trans-10,cis-12 (open bars); 9,11 = CLA cis-9,trans-11 (shaded bars). Means \pm SEM not sharing the same letter are significantly different $(P<0.05)$. 
Table 5. Effects of low-dose CLA supplementation throughout culture on production of in vitro fertilized embryos (experiment 2$)^{1}$

\begin{tabular}{lccc}
\hline Group & $\begin{array}{c}\text { Oocytes/ } \\
\text { group }\end{array}$ & $\begin{array}{c}\text { Cleavage } \\
\text { rate }(\%)\end{array}$ & $\begin{array}{c}\text { Blastocyst } \\
\text { rate }^{2}(\%)\end{array}$ \\
\hline Control & $40.5 \pm 1.6$ & $86.9 \pm 1.5$ & $38.2 \pm 2.3^{\mathrm{a}}$ \\
trans-10, cis-12, $15 \mu M$ & $42.5 \pm 1.8$ & $89.6 \pm 1.8$ & $36.6 \pm 4.8^{\mathrm{a}}$ \\
trans-10, cis-12, $25 \mu M$ & $42.8 \pm 1.7$ & $82.4 \pm 3.0$ & $25.5 \pm 2.1^{\mathrm{b}}$ \\
cis-9, trans-11, $15 \mu M$ & $42.3 \pm 2.0$ & $83.8 \pm 1.9$ & $38.2 \pm 2.3^{\mathrm{a}}$ \\
cis-9,trans-11, $25 \mu M$ & $41.8 \pm 1.5$ & $82.1 \pm 3.9$ & $32.9 \pm 2.4^{\mathrm{ab}}$ \\
\hline
\end{tabular}

${ }^{\mathrm{a}, \mathrm{b}}$ Means within a column not sharing the same letter are significantly different $(P<0.05)$.

${ }^{1}$ Results are means \pm SEM with 4 replicates.

${ }^{2}$ Blastocyst rates are based on percentages of the original oocyte number.

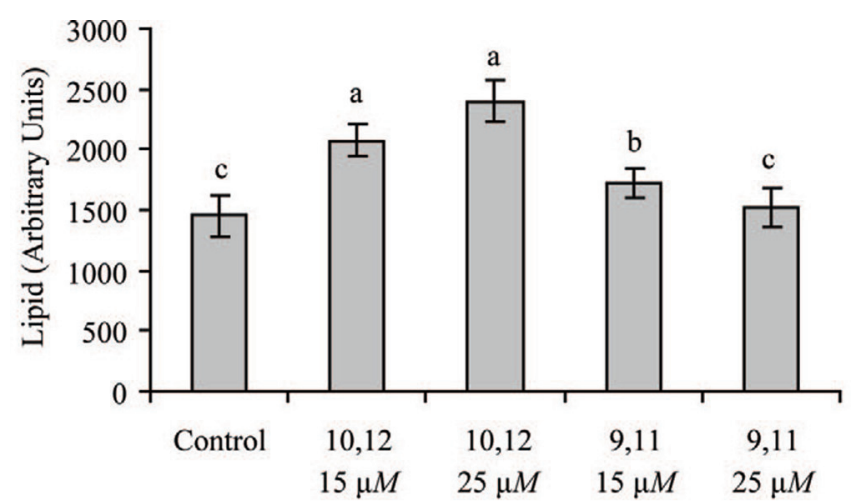

(a)

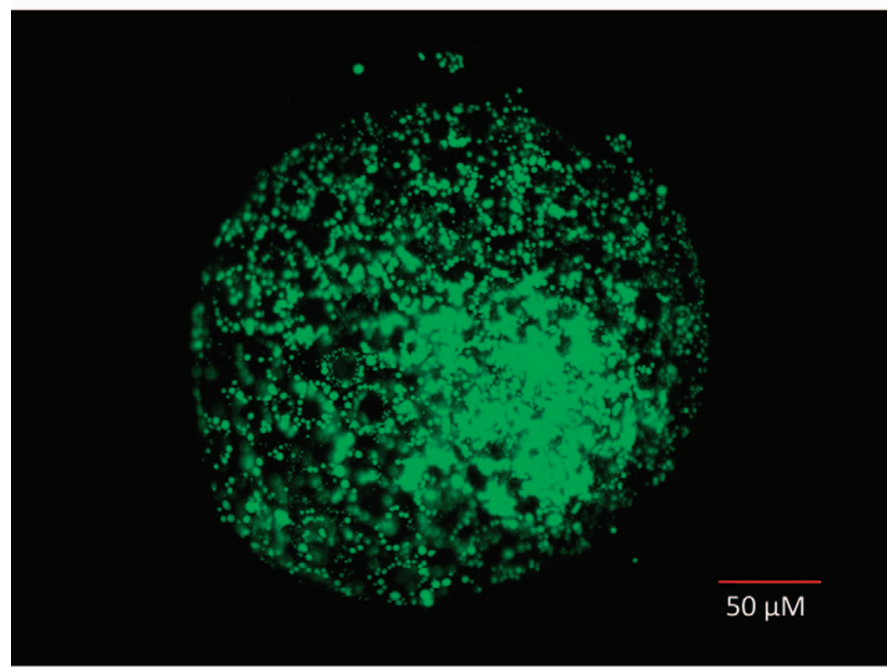

(b)

Figure 2. Effect of CLA supplementation throughout culture on lipid content in d-8 in vitro fertilized (IVF) embryos. (a) Nile red staining was used to compare lipid content among groups; $10,12=$ CLA trans-10,cis-12; 9,11 = CLA cis-9,trans-11. Means \pm SEM not sharing the same letter are significantly different $(P<0.05)$. (b) Representative embryo stained with Nile red at d 8 postinsemination. for groups treated with either CLA isomer at $15 \mu M$, blastomere counts did not differ $(P>0.05)$ among treatment groups $(118 \pm 15,133 \pm 16,112 \pm 17,123$ \pm 15 , and $119 \pm 16$ for control; $t 10, c 12$ at $15 \mu M$ IVM; $t 10, c 12$ at $15 \mu M$ IVC; $c 9, t 11$ at $15 \mu M$ IVM; and $c 9, t 11$ at $15 \mu M$ IVC; respectively).

\section{Experiment 4}

Although we found some variation in embryo cleavage rates among groups, overall percentages were within an acceptable range (86 to $93 \%$; Table 7 ). The numbers of embryos and blastocyst rate percentage were similar among treatment groups. Analyses for blastocyst post-rewarming survival rates showed no statistical differences among all vitrified groups, except for $t 10, c 12$ $15 \mu M$ IVM, which yielded the lowest survival values. Analyses of blastomere cell counts showed that embryos treated with $100 \mu M c 9, t 11$ before vitrification had the highest blastomere count among all vitrified groups $(P$ $<0.01$; Figure 4) with cell counts comparable $(P>$ 0.05 ) to that in the nonvitrified control group (Control+).

\section{DISCUSSION}

The main objective of the current study was to assess the effects of supplementation with CLA isomers at different stages during the in vitro embryo production process on maturation, cleavage, and preimplantation blastocyst development. In the first experiment, parthenogenetic activation of oocytes was performed, rather than IVF, to restrict the potential effects of CLA treatment to oocytes and embryos without confounding actions on sperm or fertilization. Supplementation with either $t 10, c 12$ or $c 9, t 11$ during IVM had no effect on maturation, cleavage, or blastocyst rates. However, treatment with high doses of these isomers during the entire IVC period resulted in significant differences in maturation and cleavage rates, which were mixed (positive and negative), and decreased blastocyst rates that 
Table 6. Effects of low-dose CLA supplementation during in vitro maturation (IVM) or throughout the entire in vitro embryo production (IVC) period (experiment 3$)^{1}$

\begin{tabular}{lccc}
\hline Group & $\begin{array}{c}\text { Oocytes/ } \\
\text { group }\end{array}$ & $\begin{array}{c}\text { Cleavage } \\
\text { rate }(\%)\end{array}$ & $\begin{array}{c}\text { Blastocyst } \\
\text { rate }^{2}(\%)\end{array}$ \\
\hline Control & $45.3 \pm 1.9$ & $90.1 \pm 0.2$ & $34.2 \pm 1.2^{\mathrm{a}}$ \\
trans-10, cis-12, $15 \mu M$ IVM & $44.8 \pm 2.8$ & $92.3 \pm 1.0$ & $28.6 \pm 1.2^{\mathrm{b}}$ \\
trans-10, cis-12, 15 $\mu$ M IVC & $44.5 \pm 2.6$ & $87.1 \pm 3.2$ & $20.6 \pm 2.4^{\mathrm{c}}$ \\
cis-9, trans-11, 15 $\mu M$ IVM & $45.0 \pm 2.3$ & $88.4 \pm 2.4$ & $34.0 \pm 1.6^{\mathrm{a}}$ \\
cis-9,trans-11, 15 $\mu M$ IVC & $46.0 \pm 3.0$ & $90.2 \pm 1.1$ & $27.7 \pm 1.2^{\mathrm{b}}$ \\
\hline
\end{tabular}

${ }^{a-c}$ Means within a column not sharing the same letter are significantly different $(P<0.05)$.

${ }^{1}$ Results are means \pm SEM with 4 replicates.

${ }^{2}$ Blastocyst rates are based on percentages of the original oocyte number.

Table 7. The effect of CLA supplementation during in vitro maturation of oocytes (IVM) or for $36 \mathrm{~h}$ late in embryo culture (IVC) on embryo survival rates following vitrification, rewarming, and culture for $24 \mathrm{~h}$ (experiment 4$)^{1}$

\begin{tabular}{|c|c|c|c|c|c|}
\hline Group & $\begin{array}{c}\text { Oocytes/ } \\
\text { group }\end{array}$ & $\begin{array}{l}\text { Cleavage } \\
\text { rate }(\%)\end{array}$ & $\begin{array}{c}\text { Embryos }^{2} \\
\text { (no.) }\end{array}$ & $\begin{array}{c}\text { Blastocyst } \\
\text { rate }(\%)\end{array}$ & $\begin{array}{l}\text { Survival } \\
\operatorname{rate}^{3}(\%)\end{array}$ \\
\hline $\begin{array}{l}\text { Control } \\
\text { trans-10, cis-12, } 15 \mu M \mathrm{IVM}^{4} \\
\text { trans-10,cis-12, } 100 \mu M \mathrm{IVC}^{5} \\
\text { cis-9, trans-11, } 15 \mu M \mathrm{IVM}^{4} \\
\text { cis-9,trans-11, } 100 \mu M \mathrm{IVC}^{5}\end{array}$ & $\begin{array}{l}42.0 \pm 0.0 \\
42.5 \pm 0.5 \\
40.0 \pm 2.0 \\
42.5 \pm 0.5 \\
40.5 \pm 0.5\end{array}$ & $\begin{array}{l}90.5 \pm 2.3^{\mathrm{ab}} \\
92.9 \pm 0.1^{\mathrm{a}} \\
86.3 \pm 0.5^{\mathrm{b}} \\
90.6 \pm 0.1^{\mathrm{ab}} \\
86.4 \pm 1.4^{\mathrm{b}}\end{array}$ & $\begin{array}{l}14.9 \pm 2.0 \\
15.3 \pm 2.2 \\
17.5 \pm 0.9 \\
16.3 \pm 2.9 \\
15.4 \pm 3.4\end{array}$ & $\begin{array}{l}34.3 \pm 4.8 \\
35.9 \pm 5.5 \\
43.7 \pm 0.1 \\
38.2 \pm 6.3 \\
37.8 \pm 7.8\end{array}$ & $\begin{aligned} 74.8 & \pm 13.7^{\mathrm{a}} \\
9.6 & \pm 1.9^{\mathrm{b}} \\
51.3 & \pm 3.7^{\mathrm{ab}} \\
40.3 & \pm 19.4^{\mathrm{ab}} \\
82.1 & \pm 17.9^{\mathrm{a}}\end{aligned}$ \\
\hline
\end{tabular}

${ }^{\mathrm{a}, \mathrm{b}}$ Means within a column not sharing the same letter are significantly different $(P<0.05)$.

${ }^{1}$ Results are means \pm SEM with 2 replicates. Control+ (nonvitrified group) not shown.

${ }^{2}$ Number of blastocysts per replicate.

${ }^{3}$ Survival rates are based on the numbers of vitrified embryos.

${ }^{4}$ CLA supplemented only at IVM. The remainder of the culture period was performed using CLA-free medium.

${ }^{5}$ CLA supplemented on d 6.5 postfertilization for a period of $36 \mathrm{~h}$.

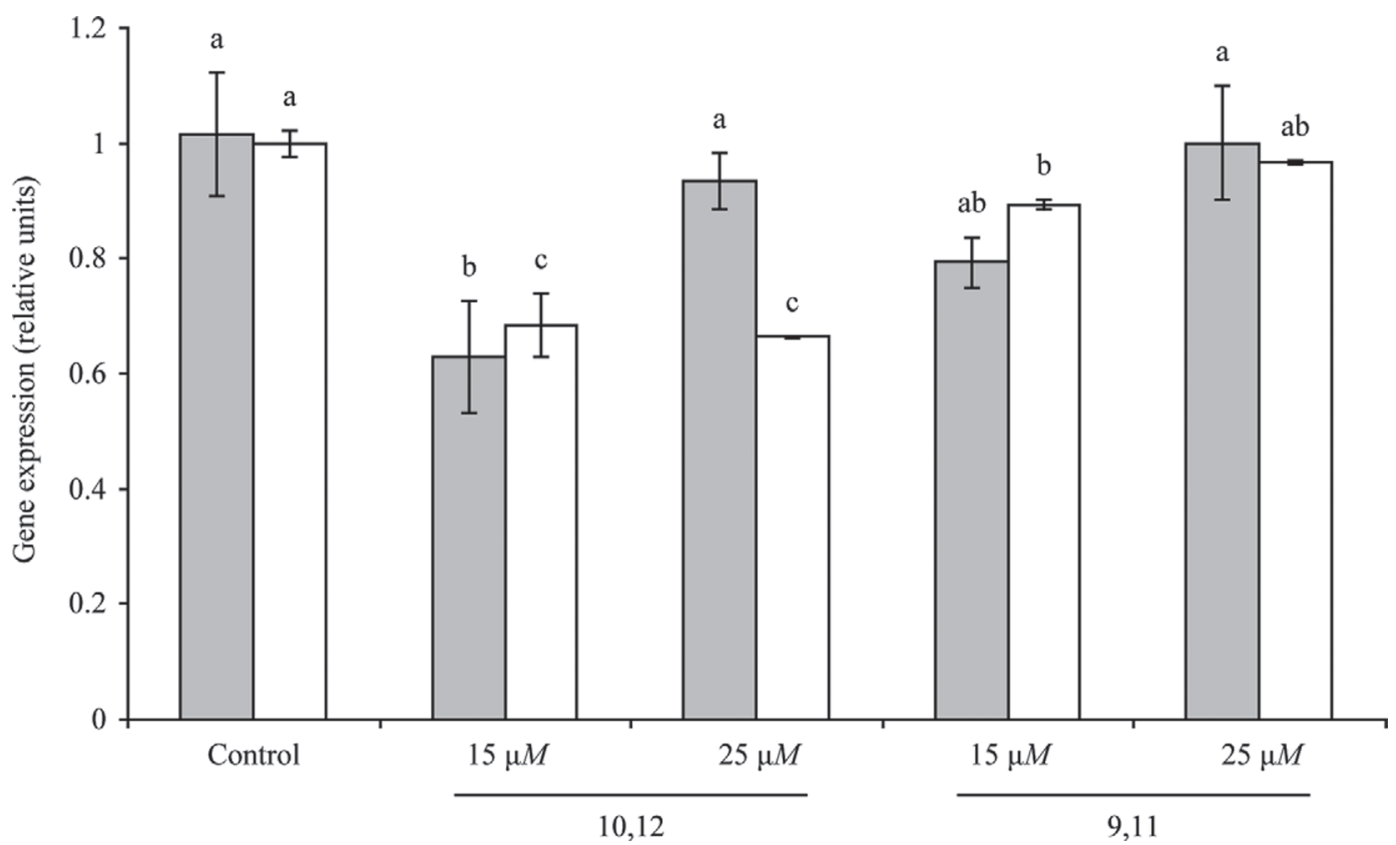

Figure 3. Effect of CLA supplementation throughout culture on expression of HSPA1A gene (shaded bars) and BIRC5 gene (open bars) in d 8 bovine in vitro fertilized embryos. 10,12 = CLA trans-10, cis-12; 9,11 = CLA cis-9,trans-11. Values represent means \pm SEM $(\mathrm{n}=13 \pm 4$ embryos per group) with statistical differences $(P<0.05)$ from control indicated by different letters. 


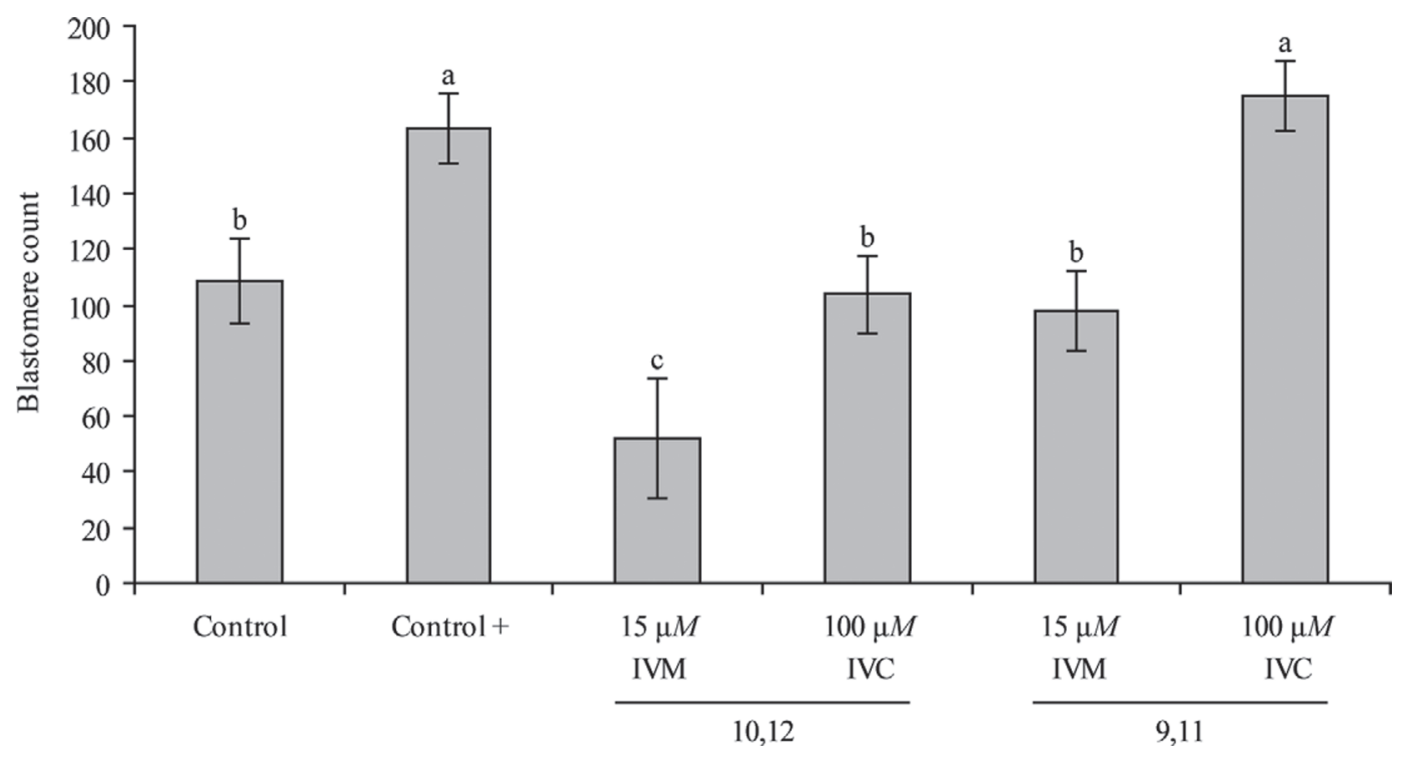

Figure 4. Effect of CLA supplementation on blastomere counts in embryos after vitrification and rewarming or in the nonvitrified control group (Control+). Embryo cultures were supplemented with $15 \mu M$ CLA isomers at in vitro maturation (IVM) or with $100 \mu M$ CLA isomers for $36 \mathrm{~h}$ (IVC) before vitrification and rewarming on d 8. 10,12 = CLA trans-10,cis-12; 9,11 = CLA cis-9, trans-11. Mean \pm SEM blastocyst cell numbers not sharing the same letter are significantly different $(P<0.05 ; \mathrm{n}=180$ total embryos across groups).

were dose dependent. The negative effects of each CLA isomer are consistent with a recent report using similar dose levels of CLA isomers (Stinshoff et al., 2014). Our subsequent trials with low CLA concentrations showed neither statistically significant differences nor benefits of supplementation on embryo development after parthenogenetic activation. Across trials (experiment 1), treatment with 15 to $100 \mu M t 10, c 12$ increased cleavage rates but with concomitant decreases in blastocyst rates that we cannot completely explain.

The addition of high doses of either isomer during IVM increased progesterone levels in media in a dose-dependent manner, which in turn was inversely related to embryo development rate (experiment 1). Previously, contradictory effects of progesterone on oocyte and embryo developmental competence have been reported (Fair and Lonergan, 2012). For example, one study indicated a detrimental effect of progesterone on in vitro-matured oocytes (Fukui et al., 1982) contrasting with others reporting a beneficial effect of progesterone during embryo culture (Pereira et al., 2009; Ferguson et al., 2012). The fact that progesterone was upregulated by the effects of each CLA isomer on cumulus cells (enclosing the oocytes) has not been reported previously. Given that the oocyte and cumulus cells are considered a complex, we suggest that CLA isomers exerted a global effect upon COC. One potential explanation may be that CLA affect intracellular signaling transducers such as Sma and Mad-related (SMAD) that are present in the cumulus cells thereby altering sterol and progesterone synthesis (Gilchrist, 2011; Salhab et al., 2011). Alternatively, CLA isomers may have affected the morphogenetic gradient generated by oocyte-secreted factors that in turn maintain cumulus cell phenotype and other functions related to sterol synthesis (Gilchrist, 2011).

Given that high CLA doses exerted detrimental effects on parthenogenetic embryos (experiment 1), we tested only the effects of low CLA concentrations in the subsequent experiments with IVF-produced embryos. Supplementation with low doses of each CLA isomer throughout IVC yielded no significant differences in terms of cleavage rates; however, concentrations greater than $15 \mu M$ resulted in a significant reduction in blastocyst rates, particularly when using the $t 10, c 12$ isomer (experiments 2 and 3). Although we observed lower blastocyst rates for each CLA isomer after treatment during either IVM or IVC compared with control, $c 9, t 11$ had a less detrimental effect on blastocyst rates than did t10,c12 (experiment 3 ). In this regard, the most consistent observation throughout our study was that long-term supplementation with 25 to $200 \mu M$ $t 10, c 12$ or $c 9, t 11$ isomers was detrimental to embryo development. Overall, these negative results with CLA isomers confirm another report using a serum-free culture system for bovine embryos (Stinshoff et al., 2014). The current findings are also in agreement with Hughes et al. (2011), who found that long-term culture in the presence of other PUFA resulted in poor quality embryos. It has been reported that high levels of FA may 
affect mitochondrial activity and downstream cell signaling during in vitro embryo production, as reflected by compromised embryo development (Marei et al., 2009; Al Darwich et al., 2010; Van Hoeck et al., 2013).

Considering further the effects on embryo lipid metabolism, Al Darwich et al. (2010) showed that supplementation of bovine embryo medium with several PUFA, including CLA $t 10, c 12$, regulated mRNA expression of target genes for lipid synthesis. Regulation of the activity of enzymes was suggested to modify the balance of saturated:unsaturated FA in blastocysts that contributes to or interferes with membrane fluidity. The CLA $t 10, c 12$ treatment caused a significant downregulation of fatty acid desaturase 2 (FADS2). More recently, Stinshoff et al. (2014) studied the effects of each of the CLA isomers on mRNA abundance of target gene transcripts related to fat metabolism. Although interaction with the effects of the carrier solvent (dimethyl sulfoxide) likely masked the magnitude, $50 \mu M t 10, c 12$ or $c 9, t 11$ downregulated acetyl-CoA acyltransferase 1, fatty acid synthase (FAS), carnitine palmitoyltransferase 2, and stearoyl-coenzyme A desaturase to influence embryonic lipid metabolism.

In contrast to the previous effects of CLA isomers on gene transcripts for lipid metabolism and contrary to our expectations, treatment of embryos with low doses of $t 10, c 12$ yielded a greater epifluorescent signal for lipid content. Based upon the work of Granlund et al. (2005), we speculate that a glucose transporter could be affected by $t 10, c 12$, thus exerting its effects on lipogenic transcription factors such as liver $\mathrm{X}$ receptor $\alpha(\mathrm{LXR} \alpha)$. Besides being involved in its own upregulation, liver $\mathrm{X}$ receptor $\alpha$ is involved in the regulation of other lipogenic genes such as sterol regulatory elementbinding transcription factor 1 and FAS. Another possible scenario is that CLA isomers, especially $t 10, c 12$, may redirect glucose toward the triglyceride synthesis pathway, thus compromising glycolysis, the metabolic pathway that is physiologically enhanced during morula compaction (Thompson et al., 2000; Absalón-Medina et al., 2014). If this is true, the redirection of glucose fate and increased lipogenesis resulting in increased lipid content may have been detrimental for embryo development and further aggravated by lesser metabolism of internal lipids due to the lack of L-carnitine in vitro (Sutton-McDowall et al., 2012). In the current study, the relative transcript abundance of 2 antiapoptotic markers whose expression is related to embryo survival (BIRC5 and HSPA1A) was reduced by low $t 10, c 12$ concentrations, whereas supplementation with $c 9, t 11$ maintained relative expression of these genes similar to that in the control group. From this evidence, we report that $t 10, c 12$ was consistently detrimental not only to blastocyst rates, but also to developmental performance, as evidenced by antiapoptotic markers. Although the detrimental effect of increased lipid accumulation in bovine embryos cultured in the presence of serum was reported previously (Abe et al., 2002; Reis et al., 2003), serum-free culture may affect the metabolic profile of embryos, because they may rely on internal lipid stores as a source of ATP. The extent to which the embryo optimally relies on lipids remains unclear and is an important area of investigation toward development of serum-free defined culture systems.

Cytoplasmic lipid content of oocytes or embryos from bovine and other species such as equine, swine, and canine is physiologically higher than that in human and murine species (Sturmey et al., 2009). Because excessive lipid accumulation affects the developmental performance and survival of embryos undergoing cryopreservation procedures (Abe et al., 2002; Pereira et al., 2007), we tested the ability of embryos to survive cryopreservation following supplementation with either the low, $15 \mu M$, CLA dose during IVM or with a 100 $\mu M$ dose for a short period late in IVC. The higher dose was tested in previous studies (Pereira et al., 2007; Al Darwich et al., 2010). In our study, it was apparent that embryos treated with $15 \mu M$ t10,c12 during IVM were less viable quantitatively (i.e., reduced blastocyst survival rates and blastomere cell counts). Shu et al. (2009) reported a subjective blastomere loss in human embryos postthaw due to the cryopreservation process itself (slow freezing). A marked reduction in blastomere count following vitrification and rewarming only occurred for embryos exposed to $t 10, c 12$ during IVM, the same treatment group that had the lowest percentage survival. This strong association suggests that the loss of blastomeres for this group was likely the result of decreased viability and greater sensitivity to cryopreservation-induced damage.

Previous studies supplementing culture media with CLA $t 10, c 12$ in preparation for testing embryo sensitivity to cryopreservation have yielded contradictory results. Enhanced viability and cryosurvival of bovine embryos after thawing and culture were demonstrated in earlier reports (Pereira et al., 2007, 2008), but a more recent report found lower embryo survival using the same concentration of $t 10, c 12$ (Al Darwich et al., 2010). In those studies, $t 10, c 12$ was supplemented as the free FA, and embryos were incubated in the presence of serum during the entire culture, whereby cytoplasmic lipid accumulation was expected to become greater. Although their cryopreservation procedures differed, it is unclear whether this could account for the contradictory outcomes on embryo survival after thawing. In the current study, the CLA isomers were conjugated to FA-free BSA, reportedly the most efficient carrier for PUFA to be delivered successfully (Hughes et al., 2011). 
A vitrification procedure was used for cryopreservation of blastocysts (similar to that in Al Darwich et al., 2010).

Short-term supplementation with $t 10, c 12$ or $c 9, t 11$ isomer for $36 \mathrm{~h}$ before vitrification did not alter embryo survival rates after rewarming and culture. However, one of the most important findings in the current study was that short-term incubation with $100 \mu M c 9, t 11$ from morula to early blastocyst stage resulted in increased blastomere counts after rewarming and culture that were equivalent to those of the quality control group $($ Control+) not undergoing vitrification. These beneficial effects of short-term supplementation with $c 9, t 11$ are most likely explained by improved membrane fluidity (Zeron et al., 2002; Pereira et al., 2008). A similar benefit did not occur in response to CLA t10,c12.

\section{CONCLUSIONS}

A series of experiments explored the effects of CLA isomers during the in vitro embryo production process of parthenogenetically activated or in vitro fertilized embryos up to d 8 postactivation or postfertilization stage, respectively. Overall, the effects of CLA supplementation on bovine embryo development and performance were detrimental, especially with the $t 10, c 12$ isomer and with longer periods in culture. When tested during cryopreservation procedures, embryos supplemented with $100 \mu M c 9, t 11$ for $36 \mathrm{~h}$ just before vitrification showed the highest survival rates and highest blastomere cell counts following rewarming. Additional benefits from CLA supplementation, other than better performance after rewarming of vitrified embryos, require further study.

\section{ACKNOWLEDGMENTS}

The authors thank the Consejo Nacional de Ciencia y Tecnología (CONACyT, México) as well as the Department of Animal Science (Cornell University, Ithaca, NY) for providing financial support to V. A. AbsalónMedina for the PhD program. Thanks to Cargill ( $\mathrm{Wy}-$ alusing, PA) for providing bovine ovaries on a weekly basis. We also appreciate the support from Genex Cooperative Inc. (Ithaca, NY) for donating bovine semen for our IVF trials. Access to P. Cohen's laboratory (Biomedical Sciences Department, Cornell University, Ithaca, NY) equipment and advice for epifluorescence microscopy is gratefully acknowledged. Special thanks to D. E. Bauman and V. Selvaraj (Animal Science Department, Cornell University) for their insightful comments during the development of this manuscript.

\section{REFERENCES}

Abe, H., S. Yamashita, T. Satoh, and H. Hoshi. 2002. Accumulation of cytoplasmic lipid droplets in bovine embryos and cryotolerance of embryos developed in different culture systems using serum-free or serum-containing media. Mol. Reprod. Dev. 61:57-66.

Absalón-Medina, V. A., W. R. Butler, and R. O. Gilbert. 2014. Preimplantation embryo metabolism and culture systems: Experience from domestic animals and clinical implications. J. Assist. Reprod. Genet. 31:393-409.

Al Darwich, A., C. Perreau, M. H. Petit, P. Papillier, J. Dupont, D. Guillaume, P. Mermillod, and F. Guignot. 2010. Effect of PUFA on embryo cryoresistance, gene expression and AMPKalpha phosphorylation in IVF-derived bovine embryos. Prostaglandins Other Lipid Mediat. 93:30-36.

Bastos, G. M., P. B. Goncalves, and V. Bordignon. 2008. Immunolocalization of the high-mobility group N2 protein and acetylated histone H3K14 in early developing parthenogenetic bovine embryos derived from oocytes of high and low developmental competence. Mol. Reprod. Dev. 75:282-290.

Bauman, D. E., J. W. Perfield II, K. J. Harvatine, and L. H. Baumgard. 2008. Regulation of fat synthesis by conjugated linoleic acid: Lactation and the ruminant model. J. Nutr. 138:403-409.

Bonilla, L., and P. J. Hansen. 2009. Determining lipid content in embryos using Nile red fluorescence. Accessed February 2010. http:// animal.ifas.ufl.edu/hansen/lab_protocol_docs/detection_lipids_ embryos_nile_red_fluorescence.pdf.

Bougnoux, P., N. Hajjaji, K. Maheo, C. Couet, and S. Chevalier. 2010. Fatty acids and breast cancer: Sensitization to treatments and prevention of metastatic re-growth. Prog. Lipid Res. 49:76-86.

Elrod, C. C., and W. R. Butler. 1993. Reduction of fertility and alteration of uterine $\mathrm{pH}$ in heifers fed excess ruminally degradable protein. J. Anim. Sci. 71:694-701.

Fair, T., and P. Lonergan. 2012. The role of progesterone in oocyte acquisition of developmental competence. Reprod. Domest. Anim. 47:142-147.

Ferguson, C. E., D. J. Kesler, and R. A. Godke. 2012. Progesterone enhances in vitro development of bovine embryos. Theriogenology $77: 108-114$

Fouladi-Nashta, A. A., K. E. Wonnacott, C. G. Gutierrez, J. G. Gong, K. D. Sinclair, P. C. Garnsworthy, and R. Webb. 2009. Oocyte quality in lactating dairy cows fed on high levels of n-3 and n-6 fatty acids. Reproduction 138:771-781.

Fukui, Y., M. Fukushima, Y. Terawaki, and H. Ono. 1982. Effect of gonadotropins, steroids and culture media on bovine oocyte maturation in vitro. Theriogenology 18:161-175.

Gilchrist, R. B. 2011. Recent insights into oocyte-follicle cell interactions provide opportunities for the development of new approaches to in vitro maturation. Reprod. Fertil. Dev. 23:23-31.

Granlund, L., J. I. Pedersen, and H. I. Nebb. 2005. Impaired lipid accumulation by trans10, cis 12 CLA during adipocyte differentiation is dependent on timing and length of treatment. Biochim. Biophys. Acta 1687:11-22.

Holm, P., P. J. Booth, M. H. Schmidt, T. Greve, and H. Callesen. 1999. High bovine blastocyst development in a static in vitro production system using SOFaa medium supplemented with sodium citrate and myo-inositol with or without serum-proteins. Theriogenology 52:683-700.

Hughes, J., W. Y. Kwong, D. Li, A. M. Salter, R. G. Lea, and K. D. Sinclair. 2011. Effects of omega-3 and -6 polyunsaturated fatty acids on ovine follicular cell steroidogenesis, embryo development and molecular markers of fatty acid metabolism. Reproduction 141:105-118.

Janovick-Guretzky, N. A., H. M. Dann, D. B. Carlson, M. R. Murphy, J. J. Loor, and J. K. Drackley. 2007. Housekeeping gene expression in bovine liver is affected by physiological state, feed intake, and dietary treatment. J. Dairy Sci. 90:2246-2252.

Keating, A. F., J. J. Kennelly, and F. Q. Zhao. 2006. Characterization and regulation of the bovine stearoyl-CoA desaturase gene promoter. Biochem. Biophys. Res. Commun. 344:233-240. 
Livak, K. J., and T. D. Schmittgen. 2001. Analysis of relative gene expression data using real-time quantitative PCR and the $2^{-\triangle \Delta \mathrm{CT}}$ method. Methods 25:402-408.

Lock, A. L., and D. E. Bauman. 2004. Modifying milk fat composition of dairy cows to enhance fatty acids beneficial to human health. Lipids 39:1197-1206.

Marei, W. F., D. C. Wathes, and A. A. Fouladi-Nashta. 2009. The effect of linolenic acid on bovine oocyte maturation and development. Biol. Reprod. 81:1064-1072.

Parrish, J. J., J. Susko-Parrish, M. A. Winer, and N. L. First. 1988. Capacitation of bovine sperm by heparin. Biol. Reprod. 38:11711180.

Parrish, J. J., J. L. Susko-Parrish, and N. L. First. 1989. Capacitation of bovine sperm by heparin: Inhibitory effect of glucose and role of intracellular pH. Biol. Reprod. 41:683-699.

Pereira, R. M., M. C. Baptista, M. I. Vasques, A. E. Horta, P. V. Portugal, R. J. Bessa, J. C. Silva, M. S. Pereira, and C. C. Marques. 2007. Cryosurvival of bovine blastocysts is enhanced by culture with trans-10 cis-12 conjugated linoleic acid (10t,12c CLA). Anim. Reprod. Sci. 98:293-301.

Pereira, R. M., I. Carvalhais, J. Pimenta, M. C. Baptista, M. I. Vasques, A. E. Horta, I. C. Santos, M. R. Marques, A. Reis, M. S. Pereira, and C. C. Marques. 2008. Biopsied and vitrified bovine embryos viability is improved by trans-10, cis-12 conjugated linoleic acid supplementation during in vitro embryo culture. Anim. Reprod. Sci. 106:322-332.

Pereira, R. M., and C. C. Marques. 2008. Animal oocyte and embryo cryopreservation. Cell Tissue Bank. 9:267-277.

Pereira, R. M., C. C. Marques, M. C. Baptista, M. I. Vasques, and A. E. M. Horta. 2009. Embryos and culture cells: A model for studying the effect of progesterone. Anim. Reprod. Sci. 111:31-40.

Reis, A., J. A. Rooke, G. J. McCallum, M. E. Staines, M. Ewen, M. A. Lomax, and T. G. McEvoy. 2003. Consequences of exposure to serum, with or without vitamin E supplementation, in terms of the fatty acid content and viability of bovine blastocysts produced in vitro. Reprod. Fertil. Dev. 15:275-284.

Rief, S., F. Sinowatz, M. Stojkovic, R. Einspanier, E. Wolf, and K. Prelle. 2002. Effects of a novel co-culture system on development, metabolism and gene expression of bovine embryos produced in vitro. Reproduction 124:543-556.
Rizos, D., A. Gutierrez-Adan, P. Moreira, C. O'Meara, T. Fair, A C. Evans, M. P. Boland, and P. Lonergan. 2004. Species-related differences in blastocyst quality are associated with differences in relative mRNA transcription. Mol. Reprod. Dev. 69:381-386.

Salhab, M., L. Tosca, C. Cabau, P. Papillier, C. Perreau, J. Dupont, P. Mermillod, and S. Uzbekova. 2011. Kinetics of gene expression and signaling in bovine cumulus cells throughout IVM in different mediums in relation to oocyte developmental competence, cumulus apoptosis and progesterone secretion. Theriogenology 75:90-104.

Shu, Y., J. Watt, J. Gebhardt, J. Dasig, J. Appling, and B. Behr. 2009. The value of fast blastocoele re-expansion in the selection of a viable thawed blastocyst for transfer. Fertil. Steril. 91:401-406.

Stinshoff, H., S. Wilkening, A. Hanstedt, H. Bollwein, and C. Wrenzycki. 2014. Dimethylsulfoxide and conjugated linoleic acids affect bovine embryo development in vitro. Reprod. Fertil. Dev $26: 502-510$

Sturmey, R. G., A. Reis, H. Leese, and T. McEvoy. 2009. Role of fatty acids in energy provision during oocyte maturation and early embryo development. Reprod. Domest. Anim. 44:50-58.

Susko-Parrish, J. L., M. L. Leibfried-Rutledge, D. L. Northey, V. Schutzkus, and N. L. First. 1994. Inhibition of protein kinases after an induced calcium transient causes transition of bovine oocytes to embryonic cycles without meiotic completion. Dev. Biol. 166:729-739.

Sutton-McDowall, M. L., D. Feil, R. L. Robker, J. G. Thompson, and K. R. Dunning. 2012. Utilization of endogenous fatty acid stores for energy production in bovine preimplantation embryos. Theriogenology 77:1632-1641.

Thompson, J. G., C. McNaughton, B. Gasparrini, L. T. McGowan, and H. R. Tervit. 2000. Effect of inhibitors and uncouplers of oxidative phosphorylation during compaction and blastulation of bovine embryos cultured in vitro. J. Reprod. Fertil. 118:47-55.

Van Hoeck, V., J. L. M. R. Leroy, M. Arias Alvarez, D. Rizos, A. Gutierrez-Adan, K. Schnorbusch, P. E. J. Bols, H. J. Leese, and R. G. Sturmey. 2013. Oocyte developmental failure in response to elevated nonesterified fatty acid concentrations: Mechanistic insights. Reproduction 145:33-44.

Zeron, Y., D. Sklan, and A. Arav. 2002. Effect of polyunsaturated fatty acid supplementation on biophysical parameters and chilling sensitivity of ewe oocytes. Mol. Reprod. Dev. 61:271-278. 